\title{
An Extraction Method of Weak Low-Frequency Magnetic Communication Signals Based on Multisensor
}

\author{
Chao Huang, ${ }^{1,2}$ Xin $\mathrm{Xu},{ }^{1}$ Dunge Liu, ${ }^{1,2}$ Wanhua Zhu, ${ }^{1}$ \\ Xiaojuan Zhang, ${ }^{1}$ and Guangyou Fang ${ }^{1}$ \\ ${ }^{1}$ Key Laboratory of Electromagnetic Radiation and Detection Technology, Chinese Academy of Sciences, North 4th Ring Road West, \\ Haidian District, Beijing 100190, China \\ ${ }^{2}$ Graduate University of Chinese Academy of Sciences, No. 19A, Yuquan Road, Beijing 100049, China
}

Correspondence should be addressed to Chao Huang; chaohuang0507@163.com

Received 28 January 2015; Revised 31 March 2015; Accepted 8 April 2015

Academic Editor: John N. Sahalos

Copyright (C) 2015 Chao Huang et al. This is an open access article distributed under the Creative Commons Attribution License, which permits unrestricted use, distribution, and reproduction in any medium, provided the original work is properly cited.

\begin{abstract}
It is a technical challenge to effectively remove the influence of magnetic noise from the vicinity of the receiving sensors on lowfrequency magnetic communication. The traditional denoising methods are difficult to extract high-quality original signals under the condition of low SNR (the signal-to-noise ratio). In this paper, we analyze the numerical characteristics of the low-frequency magnetic field and propose the algorithms of the fast optimization of blind source separation (FOBSS) and the frequency-domain correlation extraction (FDCE). FOBSS is based on blind source separation (BSS). Signal extraction of low SNR can be implemented through FOBSS and FDCE. This signal extraction method is verified in multiple field experiments which can remove the magnetic noise by about $25 \mathrm{~dB}$ or more.
\end{abstract}

\section{Introduction}

Because the electromagnetic wave of RF (radio frequency) attenuates in the water or on land, the application of lowfrequency (LF, $30 \mathrm{~Hz} 300 \mathrm{kHz}$ ), ultralow-frequency (ULF, $300 \sim 3000 \mathrm{~Hz}$ ), and extremely low-frequency (ELF, 3 30 Hz) electromagnetic spectrum receives widespread attention $[1$, 2]. The electromagnetic wave of low frequency is mainly used in submarine communication [2] and resource exploration [3].

Many scholars have made great efforts toward the theory of propagation of low-frequency electromagnetic field $[4,5]$. They have created magnetic communication channel models [6] and analyzed the physical and statistical characteristics of channel noise [7]. Actually, many excellent signal processing methods of RF can be extended to that of low frequency. For the engineering application, numbers of signal denoising methods are proposed, such as the matched filtering [8], the optimization of receiving antenna [9], and denoising based on the noise distribution and empirical mode decomposition [10]. However, in the case of low signal-to-noise ratio or existing complex noise components, these methods are difficult to work effectively.

In the application of low-frequency magnetic field, not only the signal waveform should be obtained, but also the original signal should be acquired for locating magnetic source [11]. Magnetic communication receivers are often affected by the surrounding noise and signal distorts seriously. In this paper, the correlation of low-frequency magnetic field is analyzed, and the algorithms of the fast optimization of blind source separation and the frequencydomain correlation extraction are proposed. The extraction of signal can be implemented through these algorithms. FOBSS can obtain the signal similar to the original signal waveform. FDCE recovers the original signal combining with FOBSS. In essence, we acquire more information (or more relationships) through multisensor comparing with single 
sensor. We associate the advantages of the algorithms and the inner relationships to achieve the signal extraction.

In this paper, contents are arranged as follows. Section 1 introduces the background of the low-frequency field application and the present situation of low-frequency magnetic signal processing methods; Section 2 introduces the basic theory of blind source separation; Section 3 analyzes the numerical characteristics of low-frequency magnetic field sources and proposes FOBSS and FDCE; Section 4 presents the experimental design process and verifies algorithms through multiple experiments; finally, Section 5 summarizes the full text.

\section{BSS}

When signal is interfered by multiple noise sources under the condition of low SNR, the methods of signal decomposition (Fourier analysis, wavelet analysis, empirical mode decomposition, etc.) are not effective. BSS [12] can work well in some cases, but the requirements of BSS must be met.

In the signal processing of multisensor, assume that $\mathbf{x}=$ $\left(x_{1}, x_{2}, \ldots, x_{n}\right)^{T}$ is the observation signal vector of $n$ sensors, and each observation element of $\mathbf{x}$ is the linear mixture of $m$ signal sources $\mathbf{s}=\left(s_{1}, s_{2}, \ldots, s_{m}\right)^{T}$. The linear model of $x_{i}$ is written as follows:

$$
x_{i}=\sum_{j=1}^{m} a_{i j} s_{j}, \quad i=1,2, \ldots, n,
$$

where $x_{i}$ is the measured signal of the $i$ th sensor. $a_{i j}$ is the unknown mixing coefficient between the $j$ th source and the $i$ th sensor. $s_{j}$ is the $j$ th signal source. Usually, $m$ and $n$ are equal. Equation (1) is expressed as matrix form:

$$
\mathbf{x}=\mathbf{A} \cdot \mathbf{s}, \quad \mathbf{A} \in\left(a_{i j}\right)^{n \times m} .
$$

Generally, before signal separation, $\mathbf{x}$ is preprocessed by spheroidizing decomposition. Blind source separation is to find the solution of inverse mixed matrix $W$ and estimate the optimal source signals. The process can be shown as

$$
\mathbf{y}(t)=\mathbf{W} \cdot \mathbf{x}(t)=\mathbf{W} \cdot \mathbf{A} \cdot \mathbf{s}(t)=\widehat{\mathbf{s}}(t) .
$$

The basic requirements should be satisfied: (1) signal sources are statistically independent of each other; (2) the rank of the covariance matrix $\mathbf{x}$ is full. The results of separation are evaluated by the coherence. The time-domain coherence function between $y_{i}$ and $s_{j}$ is given by

$$
\rho_{y_{i} s_{j}}=\frac{\operatorname{cov}\left(y_{i}(t), s_{j}(t)\right)}{\sqrt{\operatorname{cov}\left(y_{i}(t)\right) \operatorname{cov}\left(s_{j}(t)\right)}},
$$

where $\operatorname{cov}(\cdot, \cdot)$ is the covariance. The sequence and amplitude of the separated signal through BSS are uncertain. When the coherence is larger than 0.8 , signal separation is successful. High coherence denotes that the waveform similarity of the separated result and the original signal is high.

\section{Characteristics and Extraction Method of Low-Frequency Magnetic Field Signal}

3.1. The Correlation Characteristics of Low-Frequency Magnetic Field. The propagations of low-frequency and highfrequency electromagnetic field are in different ways (e.g., the conduction current mainly contributes to the low-frequency field, and the displacement current plays a main role in highfrequency field). The propagation mode of low-frequency field is extremely complex. However, the near field of lowfrequency magnetic field is approximately equivalent to the radiation field of magnetic source in electromagnetic field theory [13]. The vector potential and magnetic field excited by the changing current density source $\mathrm{J}\left(\mathbf{r}^{\prime}, t\right)$ are, respectively, written as

$$
\begin{gathered}
\mathbf{A}(\mathbf{r}, t)=\frac{\mu_{0}}{4 \pi} \int_{V^{\prime}} \frac{\mathbf{J}\left(\mathbf{r}^{\prime}, t-r / c\right)}{r} d V^{\prime}=\mathbf{A}(\mathbf{r}) e^{-j \omega t}, \\
\mathbf{B}(\mathbf{r})=\nabla \times \mathbf{A}(\mathbf{r}, t)=(\nabla \times \mathbf{A}(\mathbf{r})) e^{-j \omega t},
\end{gathered}
$$

where $\mu_{0}$ is the permeability of air, $c$ is the speed of light, and $\mathbf{A}(\mathbf{r})$ is expressed as $\mathbf{A}(\mathbf{r})=\left(\mu_{0} / 4 \pi\right) \int_{V^{\prime}}\left(\mathbf{J}\left(\mathbf{r}^{\prime}\right) e^{i k r} / r\right) d V^{\prime} . \omega$ is the frequency. $k$ is the wave number. $\mathbf{r}$ is the observation point. $\mathbf{r}^{\prime}$ is the source point. $r=\left|\mathbf{r}-\mathbf{r}^{\prime}\right|$. The frequency band of magnetic source is finite $\left(\omega_{l} \leq \omega \leq \omega_{h}\right)$. Frequency is described as discrete form through the Fourier analysis. The field of observation point $\mathbf{r}_{i}$ can be given by

$$
\mathbf{B}\left(\mathbf{r}_{i}\right)=\left(\nabla \times \mathbf{A}^{\prime}\left(\mathbf{r}_{i}\right)\right) \sum_{\omega=\omega_{l}}^{\omega_{h}} e^{-j\left(\omega t-k r_{i}\right)},
$$

where $\mathbf{A}^{\prime}\left(\mathbf{r}_{i}\right)$ is a factor related to the distance. Considering the wavelength of low-frequency field that is much longer than the distance between the observation point and the source point (the ratio of wavelength to distance tends to be infinite), the phase of (7) can be expressed as

$$
\omega t-k r_{i}=\omega t-2 \pi \frac{r_{i}}{\lambda_{\omega}} \longrightarrow \omega t,
$$

where $\lambda_{\omega}$ is the wavelength of $\omega$. So the ratio of magnetic field at different observation points is

$$
\frac{\mathbf{B}\left(\mathbf{r}_{i}\right)}{\mathbf{B}\left(\mathbf{r}_{j}\right)}=\frac{\nabla \times \mathbf{A}^{\prime}\left(\mathbf{r}_{i}\right)}{\nabla \times \mathbf{A}^{\prime}\left(\mathbf{r}_{j}\right)} .
$$

And the coherence between the magnetic field of $\mathbf{B}\left(\mathbf{r}_{i}\right)$ and $\mathbf{B}\left(\mathbf{r}_{j}\right)$ is

$$
\rho_{\mathbf{B}\left(\mathbf{r}_{i}\right) \mathbf{B}\left(\mathbf{r}_{j}\right)}=1 .
$$

In theory, the magnetic fields of different observation points are highly relevant. The covariance matrix of $\mathbf{B}\left(\mathbf{r}_{i}\right)(i=$ $1,2, \ldots, m)$ is $E\left(\mathbf{B B}^{T}\right)\left(\mathbf{B}=\left(\mathbf{B}\left(\mathbf{r}_{1}\right), \mathbf{B}\left(\mathbf{r}_{2}\right), \ldots, \mathbf{B}\left(\mathbf{r}_{m}\right)\right)^{T}\right)$. The singular value decomposition of $E\left(\mathbf{B B}^{T}\right)$ is represented as $E\left(\mathbf{B} \mathbf{B}^{T}\right)=\mathbf{Q} \Sigma \mathbf{Q}^{T}$. If the rank of matrix $\Sigma$ is full, the locations of the observation points meet the requirements of signal separation. If the rank of matrix $\Sigma$ is not full, the locations of the observation points need to be adjusted (usually, placing the sensors randomly is appropriate; they need not to be relocated). 
3.2. The Fast Optimization of Blind Source Separation. Because matrix W (see Section 2) contains $m^{2}$ unknown elements, BSS is the process of $\mathbf{W}$ rotating $\mathbf{x}$ (see also Section 2). $\mathbf{W}$ is a unit orthogonal matrix. Now there is a method that reduces the numbers of the unknown elements of $\mathbf{W}$, so the iteration time of finding $\mathbf{W}$ can be shortened in calculation. According to the law of Givens rotation transformation [14], the mathematical process is given by

$$
\mathbf{T} \boldsymbol{\xi}_{k}=\mathbf{T}_{1 m} \mathbf{T}_{1(m-1)} \cdots \mathbf{T}_{12} \boldsymbol{\xi}_{k}=\left|\boldsymbol{\xi}_{k}\right| \mathbf{e}_{j},
$$

where $\mathbf{T}_{1 i}$ is the Givens matrix, $\boldsymbol{\xi}_{k}$ is an arbitrary nonzero vector, and $\mathbf{e}_{j}$ is an arbitrary standard unit vector. If $\boldsymbol{\xi}_{k}$ is an arbitrary unit vector, there is a result: $\boldsymbol{\xi}_{k}=\mathbf{T}^{-1} \mathbf{e}_{j}$. Assume that $\mathbf{T}^{-1}=\mathbf{T}^{\prime}$ ( $\mathbf{T}^{-1}$ denotes reverse rotation). The rotation process of $\mathbf{e}$ ( $\mathbf{e}$ is a standard orthogonal basis) can be expressed as $\mathbf{T}^{\prime} \mathbf{e}=\mathbf{T}_{12}^{\prime} \mathbf{T}_{13}^{\prime} \cdots \mathbf{T}_{1 m}^{\prime}=\boldsymbol{\xi}(\boldsymbol{\xi}$ is an arbitrary orthogonal basis). So the arbitrary orthogonal matrix of $m$-dimensional space can be acquired through the rotation of a standard unit matrix, and the number of rotations is at most $m-1$ times. Therefore $\mathbf{W}$ can be expressed as $\mathbf{W}\left(\theta_{1}, \theta_{2}, \ldots, \theta_{m-1}\right)$ by $m-1$ unknown elements. The iteration time of $\mathbf{W}\left(\theta_{1}, \theta_{2}, \ldots, \theta_{m-1}\right)$ can be defined as $t\left(\theta_{1}, \theta_{2}, \ldots, \theta_{m-1}\right)\left(\theta_{i}\right.$ is the $i$ th rotation angle). The iteration time of basic BSS is defined as $t\left(w_{11}, w_{12}, \ldots, w_{i j} \ldots, w_{m m}\right)$ $\left(w_{i j}\right.$ is the element of $\left.\mathbf{W}\right)$. Under the condition of the same computational resource, the iteration time of $m-1$ unknown elements is statistically shorter than that of $m^{2}$ unknown elements. Therefore the ratio of mean iteration time between $t\left(\theta_{1}, \theta_{2}, \ldots, \theta_{m-1}\right)$ and $t\left(w_{11}, w_{12}, \ldots, w_{i j} \ldots, w_{m m}\right)$ is

$$
\frac{E\left[t\left(\theta_{1}, \theta_{2}, \ldots, \theta_{m-1}\right)\right]}{E\left[t\left(w_{11}, w_{12}, \ldots, w_{m m}\right)\right]} \approx \frac{m-1}{m^{2}} .
$$

3.3. The Frequency-Domain Correlation Extraction. FOBSS can only acquire the waveforms similar to the original signals. The recovery of original signals requires excellent algorithms of signal amplitude extraction. In signal extraction, the parameters of the adaptive filtering [15] are difficult to be adjusted and the adaptive filter cannot work effectively for nonperiodic signal. The signal extraction can be implemented in the frequency domain based on the correlation function. The advantage of frequency-domain extraction contains the process of power spectrum estimation. The correlation reflects in each frequency point. $u(t)$ and $v(t)$ are, respectively, defined as the time series of signals. $u(t)$ consists of the convolutions of various signals. $v(t)$ is the known waveform similar to a component of $u(t)$. The basic model is simply described as

$$
\begin{gathered}
u(t)=\sum_{i=1}^{n} h_{i}(t) * u_{i}(t), \\
v(t)=u_{k}(t), \quad k=1,2, \ldots, n,
\end{gathered}
$$

where different components of $u(t)$ are statistically independent and $h_{i}(t)(i=1,2, \ldots, n)$ is an unknown kernel function of linear convolution. Now in order to extract $h_{k}(t) * u_{k}(t)$ of $u(t)$, we get the cross-correlation function $R_{u v}(\tau)$ between $u(t)$ and $v(t) . R_{u v}(\tau)$ can be transformed to the corresponding frequency domain as $S_{u v}(\omega)$ by the Fourier transform. The mathematical process is described as

$$
\begin{gathered}
R_{u v}(\tau)=h_{k}(\tau) * R_{u_{k} u_{k}}(\tau), \\
S_{u v}(\omega)=h_{k}(\omega) S_{u_{k} u_{k}}(\omega), \\
h_{k}(\omega)=\frac{S_{u v}(\omega)}{S_{u_{k} u_{k}}(\omega)}=\frac{S_{u v}(\omega)}{S_{v v}(\omega)},
\end{gathered}
$$

where $S_{u v}(\omega)$ is the cross power spectrum density function between $u(t)$ and $v(t), h_{k}(\omega)$ is the Fourier transform of $h_{k}(t)$, and $S_{u_{k} u_{k}}(\omega)$ is the power spectrum density function of $u_{k}(t)$. Therefore the result of extraction is

$$
h_{k}(t) * u_{k}(t)=F^{-1}\left(h_{k}(\omega) V(\omega)\right),
$$

where $V(\omega)$ is the Fourier transform of $v(t)$. We give a deviation ratio $\lambda_{e}$ of the extracted signal and the original signal such that

$$
\lambda_{e}=\frac{|E(\widehat{z}(t)-z(t))|}{|E(z(t))|} \times 100 \%,
$$

where $z(t)$ is the original signal, $\widehat{z}(t)$ is the extracted signal, and $E(\cdot)$ is the mean value. If the mean values of $z(t)$ and $\widehat{z}(t)$ are zero, $z(t)$ and $\widehat{z}(t)$ are, respectively, replaced as the peakto-peak values $z_{p-p}(t)$ and $\widehat{z}_{p-p}(t)$.

\section{Processing of the Experimental Data}

4.1. Experiment Design. We have carried out multiple sets of magnetic communication experiment. Experimental equipment consists of the transmitter, the receivers, and the magnetic noise sources. The transmitter is an audio power amplifier AE7224 and the output impedance of the amplifier is less than $1 \times 10^{6} \Omega$. The transmitter antenna is a coil loaded by a magnetic core that it looks like a cylinder which is about 1 meter long. Besides, the diameter of antenna is 0.07 meter and the coil has 300 windings. The transmitting current is $1.5 \mathrm{~A}$. The receiving antenna is also a magnetic induced coil and its conversion factor is $100 \mathrm{mV} / \mathrm{nT}$. The $\mathrm{AD}$ of the receiver is a 24 bit converter and its LSB (Least Significant Bit) is 20 bits. The quantization noise is about $0.048 \mathrm{pT}$. The experiment sites are Kangxi Prairie in Beijing and An Guli Prairie in Hebei province, China. The scene is shown in Figure 1: the magnetic signal (the signal bandwidth is about $15 \mathrm{~Hz}$ ) is transmitted with the carrier frequency of $480 \mathrm{~Hz}$, and the transmitter is located at 100 meters in the Cartesian coordinate system; the receivers (multisensor) distribute in the range of 2 meters $\sim 4$ meters away from the original point, and the sampling rate $f_{s}$ is $2400 \mathrm{~Hz}$. The actual sampling rate should be higher than Nyquist sampling rate at a suitable value; the magnetic noise is from the car engines, and the engines are in the range of 2 meters $\sim 4$ meters away from the receivers. Magnetic noise is stochastic, and its frequency bandwidth covers the signal's bandwidth. So the signal contains a certain amount of inband noise. The received signals are intensely interfered by the magnetic noise in time domain and frequency domain. 

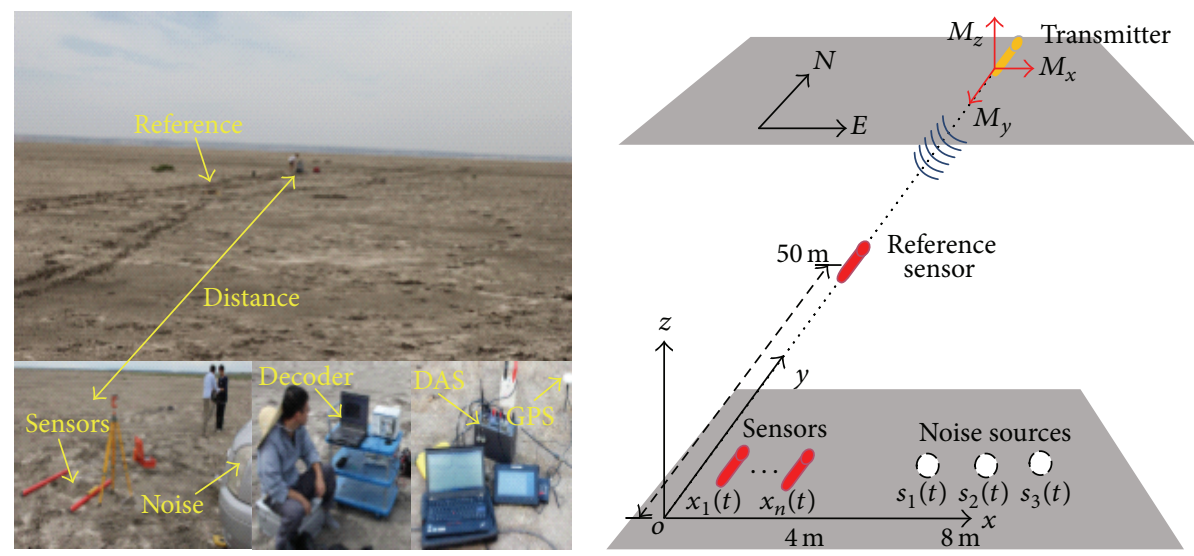

FIGURE 1: Field experiment.

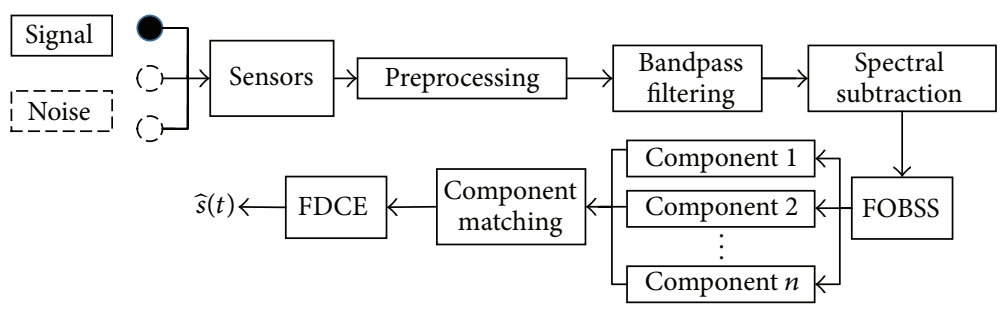

FIgURE 2: The procedure of signal extraction.

Only the signals of the multisensor close to the original point are applied to signal separation and signal extraction.

We have verified that the signal of the sensor at 50 meters is not affected by magnetic noise. When the noise sources and the transmitter are open in Figure 1, the coherence between the signal of sensor at 75 meters and that of sensor at 50 meters is 0.98 . The coherence between the signal of sensor at 75 meters and that of sensor at original point nearby is 0 . The coherence of the signal of sensor at 50 meters and that of sensor at original point nearby is 0 . But when only the transmitter is open, the coherence of different sensors is 0.98 . This phenomenon verifies that the reference sensor is not interfered by magnetic noise sources. The signal of the sensor (the reference sensor) at 50 meters is to prove the effectiveness of algorithms.

4.2. Data Processing. In Section 2, the signal model is theoretical. Under the condition of reality, the sensor itself contains micro noise, and the signal model is modified as another form:

$$
x_{i}=\sum_{j=1}^{n} a_{i j} s_{j}+N_{i}, \quad i=1,2, \ldots, n,
$$

where $a_{i j}$ is the mixing coefficient, $s_{j}$ is the $j$ th signal source, and $N_{i}$ is the micro noise of the $i$ th sensor $\left(N_{i}\right.$ is statistically independent). The procedure of signal extraction is shown in Figure 2, mainly including the preprocessing, the spectral subtraction [16], FOBSS, and FDCE. Firstly, whether the rank of the covariance matrix of the received signals is full or not should be confirmed; secondly, remove the micro noise of sensors through spectral subtraction; thirdly, signal separation is executed by FOBSS; finally, the corresponding signal waveforms are obtained by component matching, and the original signal is obtained by FDCE from the row data (the signals after band-pass filtering). The main parts are FOBSS and FDCE. Component matching is to select the required component after FOBSS (not matched filter). Besides, computing correlation function through the waveform of code is to decide which component is the required component. The required component has a similar waveform to the original signal.

Experiments are carried out by the combinations which consist in whether transmitter and magnetic noise sources are open or not, and the reference sensor at 50 meters is used to validate the correctness of algorithms. In order to illustrate data, we transform (4) to frequency domain (the frequencydomain coherence function):

$$
\rho_{y_{i} s_{j}}(\omega)=\frac{S_{y_{i} s_{j}}(\omega)}{\sqrt{S_{y_{i} y_{i}}(\omega) S_{s_{i} s_{i}}(\omega)}},
$$

where $S_{y_{i} s_{j}}(\omega)$ is the cross power spectrum density function between $y_{i}$ and $s_{j}, S_{y_{i} y_{i}}(\omega)$ is the power spectrum density function of $y_{i}$, and $S_{s_{i} s_{i}}(\omega)$ is the power spectrum density function of $s_{j}$.

As shown in Figure 1, magnetic noise sources are within the range of 2 meters 5 meters away from receivers. When only one magnetic noise is open, the coherence between the signals of different receivers is above 0.96 and there is no time delay between the signals of different receivers 


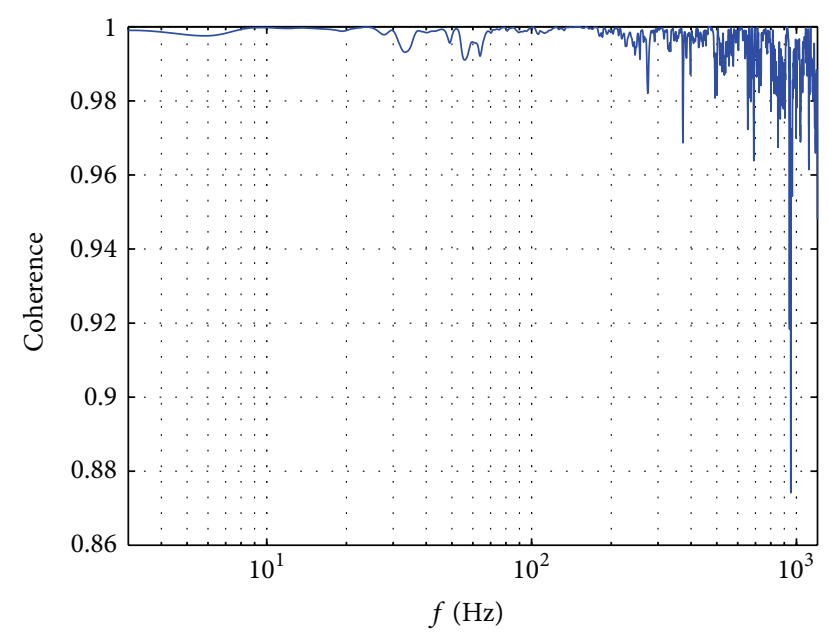

FIgURE 3: The frequency-domain coherence between magnetic noise signals of different receivers.

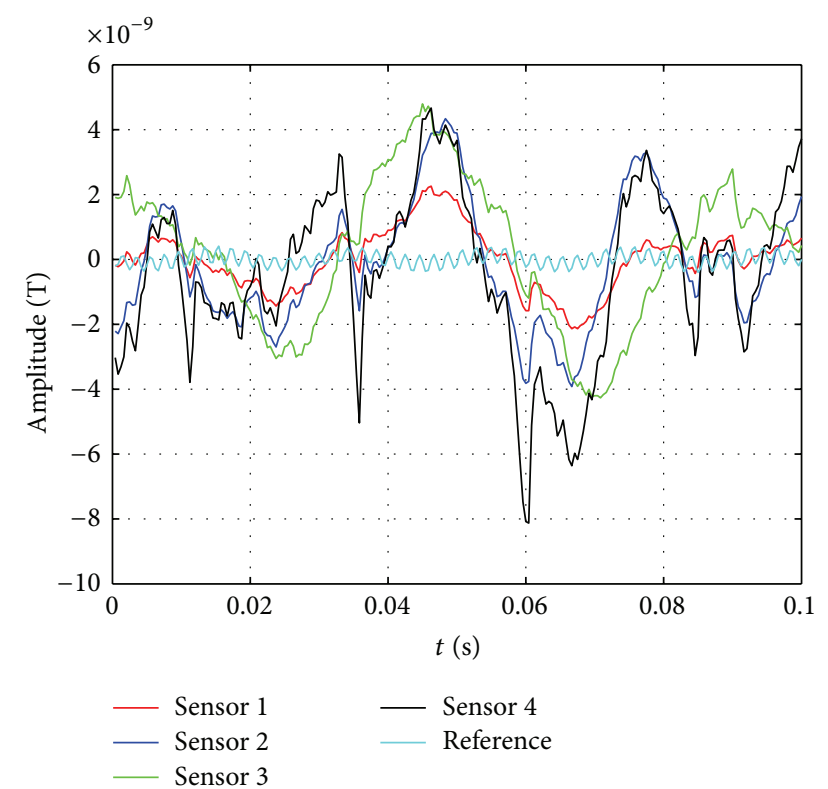

FIGURE 4: Time-domain waveforms of the received signals (three noise sources).

(these characteristics are consistent with the analysis of Section 3.1). Figure 3 shows the frequency-domain coherence, this feature meets the correlation characteristics of lowfrequency magnetic field. The reference sensor at 50 meters cannot be affected by magnetic noise, and we only use it to verify the correctness of algorithms (the reference sensor is not used for signal separation and signal extraction). The coherence between different noise sources is zero. There is no correlation between different physical sources, so components separation is possible.

When one noise source and the transmitter are open, the SNR is about $-9 \mathrm{~dB}$. When three noise sources and the transmitter are open, the SNR is about $-20 \mathrm{~dB}$. Figure 4 shows the time-domain waveforms of different receivers in the case

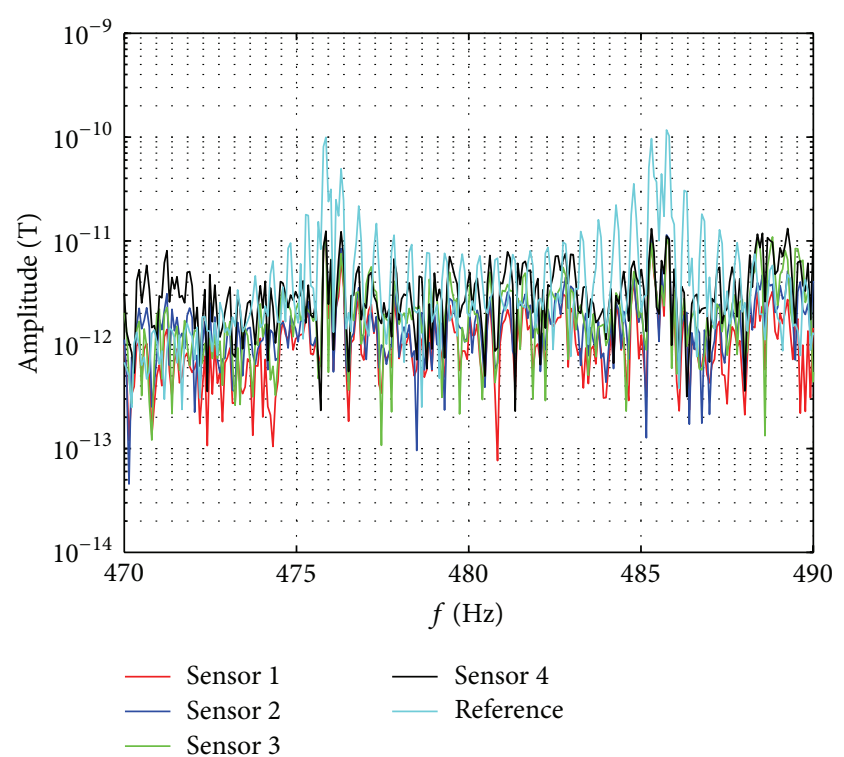

FIGURE 5: Frequency-domain waveforms of the received signals (three noise sources).

that three magnetic noise sources and the transmitter are open, and the receiver at 50 meters is not influenced by noise sources. The sequence of sensors is arranged in Figure 1. The reference sensor is at 50 meters. Figure 5 shows the frequency spectra of Figure 4. The actual locations of sensors 1, 2, 3, and 4 have a little deviation compared with the locations shown in Figure 4. According to basic electromagnetic theory, the lowfrequency magnetic field value decreases inversely proportional to distance when the source can be deemed as one point source. Because three noise sources are distributed near the sensors, three sources are not equivalent to one point source when the distance and the range of source are approximately at the same level. So field values in Figure 4 are not strictly consistent with that of one point source. There maybe exists offset when peaks and troughs appear at the same time. Since the signals are intensely interfered in time domain and frequency domain, the signal processing methods should be based on components separation but not on the synthetic filtering (it maybe contains the matched filtering, the optimal notch filtering, etc.) based on signal decomposition. The signal separation performance is generally influenced by the noise of sensor itself, industrial interference, and the experiment errors.

After preprocessing and independent noise removing, the received signals will be processed by FOBSS. We make a comparison between the separated results and the reference signal. Figure 6 shows a comparison between the separated results and reference signal (the separation results and reference signal are adjusted to the same scale), and the coherence calculated by (4) is 0.89 (in the case that three magnetic noise sources and the transmitter are open). The coherence of the separated signal under multiple sets of experiments is higher than 0.8 . The communication channel may be influenced slightly by industrial frequency interference. Since the data of Figures 6(a) and 6(b) are obtained at different time and places, 


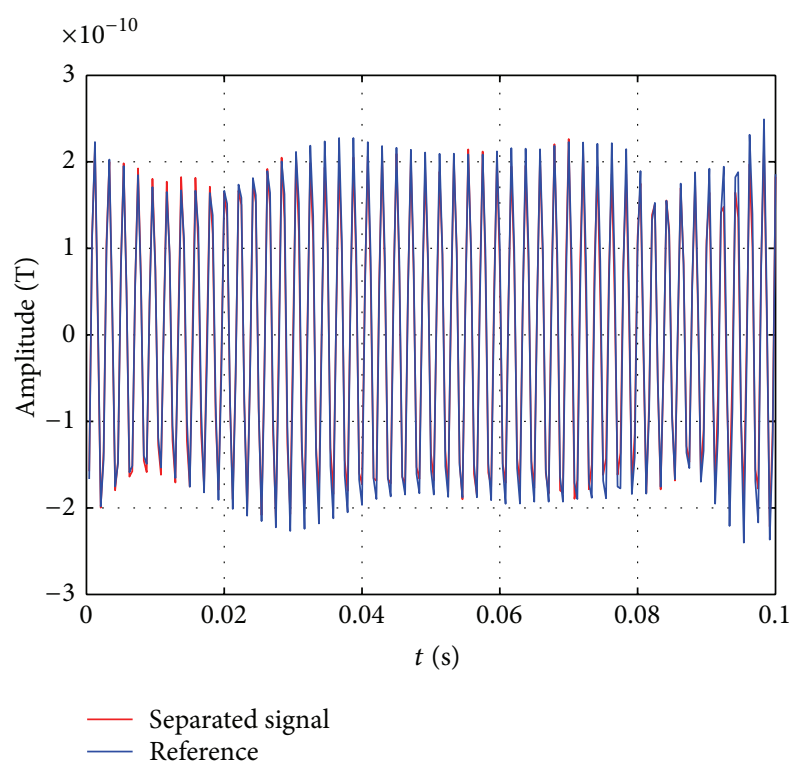

(a) The comparison of separated results in time domain (one noise source)

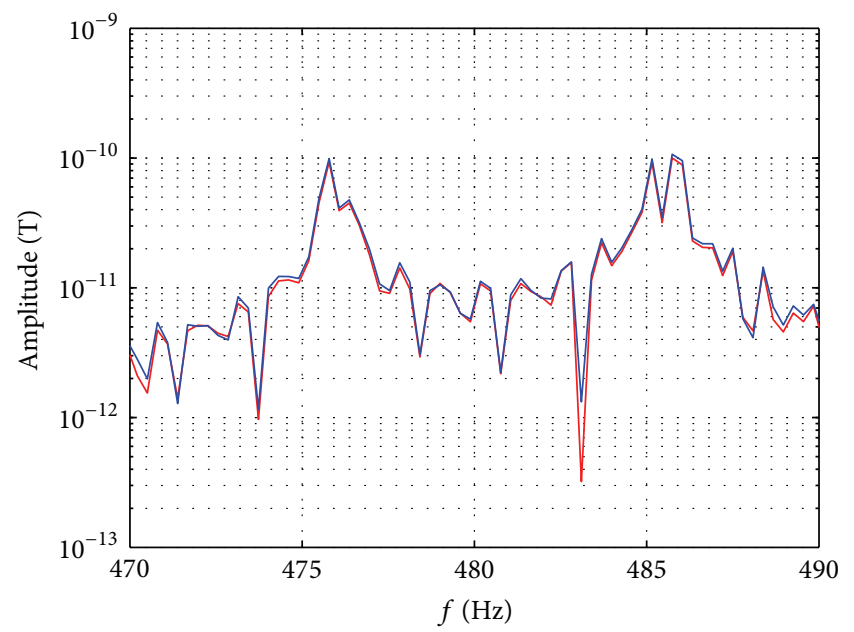

- Separated signal

- Reference

(c) The comparison of separated results in frequency domain (one noise source)

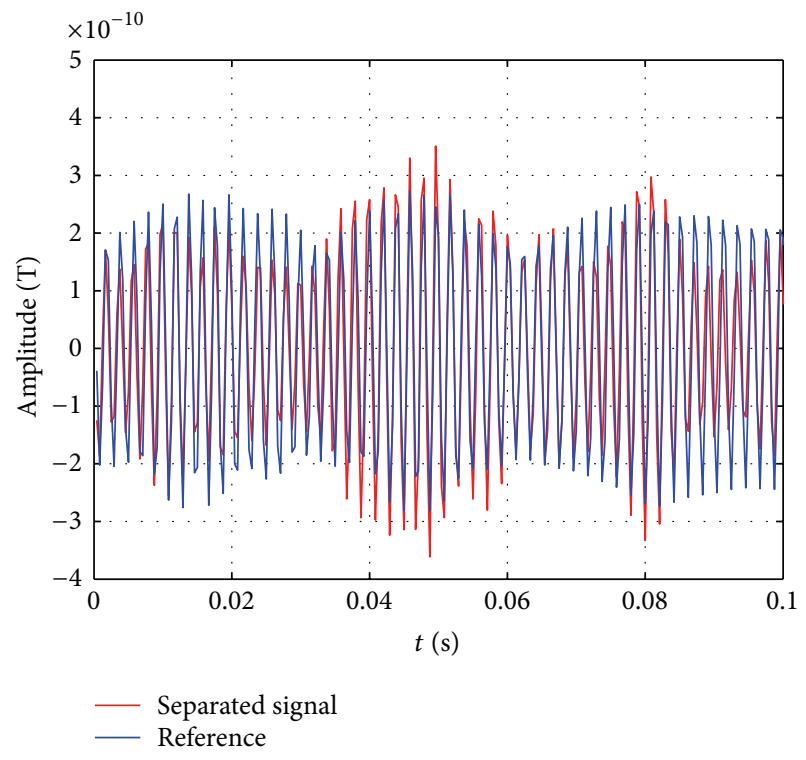

(b) The comparison of separated results in time domain (three noise sources)

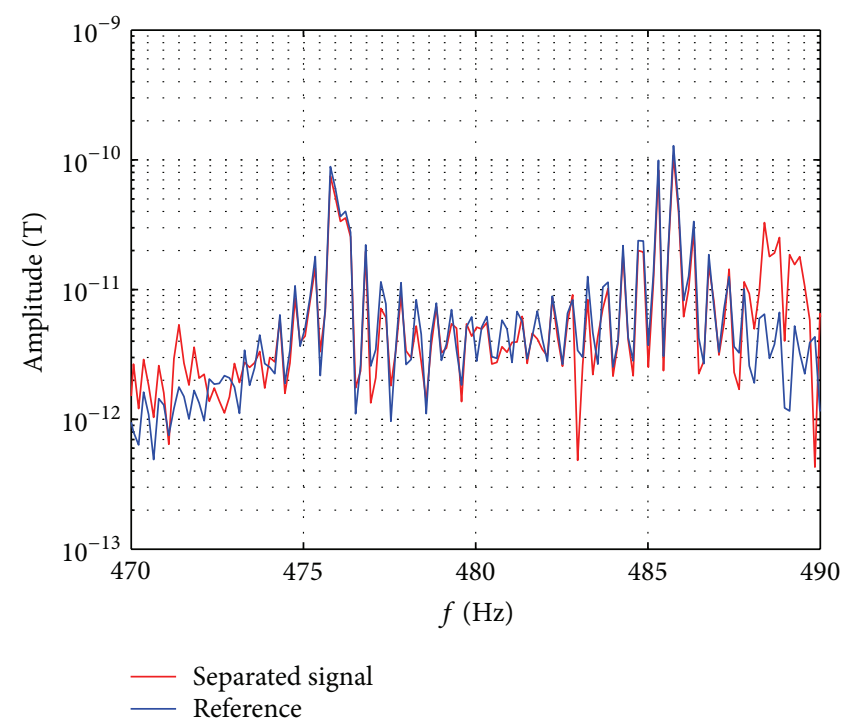

(d) The comparison of separated results in frequency domain (three noise sources)

FIGURE 6: The separated results.

it is reasonable that their envelope causes slight difference in a larger image display. Both the separated signal and the signal of the reference sensor can be decoded correctly in BPSK modulation.

The signal separation algorithm (FOBSS) can only acquire the waveforms similar to the original signals. The signal extraction is realized by (15) (FDCE). The processed results of multiple experiments are shown in Table 1; the time-domain coherence is given by (4), and the deviation ratio is given by (16).

The key purpose of this paper is to extract weak true signal masked by strong noise. The "weak" (SNR is too low) is
TABLE 1: The results of signal processing.

\begin{tabular}{lccc}
\hline Experiment site & $\begin{array}{c}\text { Number of } \\
\text { noise sources }\end{array}$ & $\begin{array}{c}\text { Time-domain } \\
\text { coherence } \rho\end{array}$ & $\begin{array}{c}\text { Deviation } \\
\text { ratio } \lambda_{e}\end{array}$ \\
\hline An Guli Prairie & 1 & 0.9924 & $3.42 \%$ \\
An Guli Prairie & 1 & 0.9533 & $4.18 \%$ \\
An Guli Prairie & 1 & 0.9501 & $4.22 \%$ \\
Kangxi Prairie & 3 & 0.8945 & $7.51 \%$ \\
Kangxi Prairie & 3 & 0.8184 & $9.03 \%$ \\
\hline
\end{tabular}

the main problem. The extracted signal through FOBSS and FDCE can be decoded errorless and it has the same waveform 


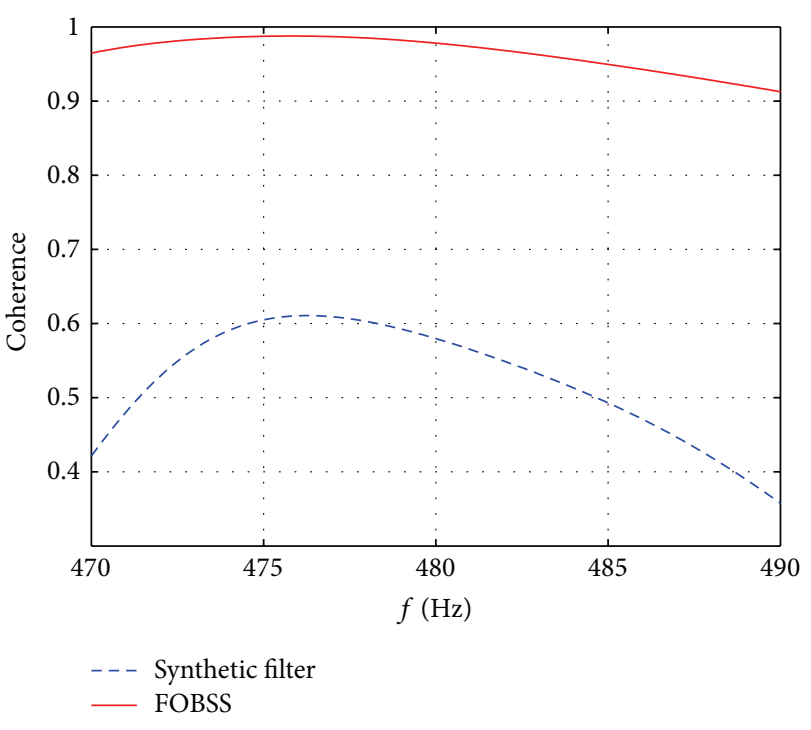

FIgURE 7: The comparison of frequency-domain coherence.

as the reference signal. They also obtained the true amplitude. In the actual environment, the signal in the transmitter has minute difference from the signal in the air. In order to verify algorithms, the reference signal only can be the signal in the air but not that in the transmitter. Even though there is a slight influence factor, both the receiving end and the reference at 50 meters are affected (other algorithms can easily solve usually). The effectiveness cannot be influenced.

4.3. Data Analysis. The coherence larger than 0.8 indicates high correlation in statistical theory. The synthetic filtering based on the time-frequency domain is difficult to filter out in-band noise; the ability of empirical mode decomposition to overcome in-band noise is limited and it is difficult to identify the mode; when the situation is more complex, it needs signal separation through building conditions. We regard the similarity (the frequency-domain coherence) to the reference signal as the standard to evaluate the ability of different signal processing methods. There is a comparison between FOBSS and the synthetic filter in Figure 7. The coherence of FOBSS tends to 1 , and the coherence of the synthetic filter is low. Simultaneously, because design of standard narrowband filter is not easy, the synthetic filter is difficult to solve the problem.

The signal preprocessing and independent noise removal are important work. Compared with basic BSS, FOBSS can effectively improve the computation time in multiple experiments. Even though (12) is a statistical result, FOBSS is more suitable for engineering application. In the original signal extraction, the deviation ratio of FDCE is about 6 percent. In short, according to the performance of FOBSS and FDCE, the proposed signal extraction method is effective.

\section{Conclusion}

In this paper, we have analyzed the characteristics of lowfrequency magnetic field and have proposed FOBSS and
FDCE. FOBSS improves processing efficiency. Even though FDCE is essentially from Fourier transform, it can be simply implemented and works well. A systemic method of extraction has been formed through the combination of FOBSS and FDCE.

This method has been effectively verified in multiple field experiments. Its advantage is that it can extract the waveform and the true amplitude under the condition of low SNR. It can remove the magnetic noise by about $25 \mathrm{~dB}$ or more. In future work, we will study the statistic characteristics of magnetic noise and give the mathematical relationship between the performance of the algorithms and independent noise.

Weak signal extraction is a difficult problem in signal processing. For the engineering application of low-frequency magnetic field, this paper has made preliminary progress in narrowband signal processing. In order to expand the application range of low-frequency signal, such as oil exploration and mining exploration, we need to increase the signal bandwidth. Because the processing of wideband signal has more difficulties, we will make further study.

\section{Conflict of Interests}

The authors declare that there is no conflict of interests regarding the publication of this paper.

\section{Acknowledgments}

This research is sponsored by the National High Technology Research and Development Program of China (Grant no. 2014AA093407) and the National Natural Science Foundation of China (Grant no. 41374186).

\section{References}

[1] A. Abubakar, T. M. Habashy, V. L. Druskin, L. Knizhnerman, and D. Alumbaugh, " $2.5 \mathrm{D}$ forward and inverse modeling for interpreting low-frequency electromagnetic measurements," Geophysics, vol. 73, no. 4, pp. F165-F177, 2008.

[2] S. A. Wolf, J. R. Davis, and M. Nisenoff, "Superconducting extremely low frequency (ELF) magnetic field sensors for submarine communications," IEEE Transactions on Communications, vol. 22, no. 4, pp. 549-554, 1974.

[3] R. E. Grimm, "Low-frequency electromagnetic exploration for groundwater on Mars," Journal of Geophysical Research: Planets, vol. 107, no. E2, p. 1, 2002.

[4] W. Pan and K. Li, Propagation of SLF/ELF Electromagnetic Waves, Springer, 2014.

[5] L. O. Løseth, H. M. Pedersen, B. Ursin, L. Amundsen, and S. Ellingsrud, "Low-frequency electromagnetic fields in applied geophysics: waves or diffusion?" Geophysics, vol. 71, no. 4, pp. W29-W40, 2006.

[6] H. E. Rowe, "Extremely low frequency (ELF) communication to submarines," IEEE Transactions on Communications, vol. 22, no. 4, pp. 371-385, 1974.

[7] E. C. Field Jr. and M. Lewinstein, "Amplitude-probability distribution model for VLF/ELF atmospheric noise," IEEE Transactions on Communications, vol. 26, no. 1, pp. 83-87, 1978.

[8] J. E. Evans and A. S. Griffiths, "Design of a sanguine noise processor based upon world-wide extremely low frequency (ELF) 
recordings," IEEE Transactions on Communications, vol. 22, no. 4, pp. 528-539, 1974.

[9] P. R. Bannister, "Orbiting transmitter and antenna for spaceborne communications at ELF/VLF to submerged submarines," in AGARD, ELF/VLF/LF Radio Propagation and Systems Aspects 14 p (SEE N93-30727 11-32), vol. 1, 1993.

[10] Y. Jeng, M.-J. Lin, C.-S. Chen, and Y.-H. Wang, "Noise reduction and data recovery for a VLF-EM survey using a nonlinear decomposition method," Geophysics, vol. 72, no. 5, pp. F223F235, 2007.

[11] A. P. Nickolaenko and I. G. Kudintseva, "A modified technique to locate the sources of ELF transient events," Journal of Atmospheric and Terrestrial Physics, vol. 56, no. 11, pp. 14931498, 1994.

[12] M. T. Akhtar, T.-P. Jung, S. Makeig, and G. Cauwenberghs, "Recursive independent component analysis for online blind source separation," in Proceedings of the IEEE International Symposium on Circuits and Systems (ISCAS '12), pp. 2813-2816, IEEE, May 2012.

[13] C. H. Papas, Theory of Electromagnetic Wave Propagation, Courier Dover Publications, 2013.

[14] F. Ling, "Givens rotation based least squares lattice and related algorithms," IEEE Transactions on Signal Processing, vol. 39, no. 7, pp. 1541-1551, 1991.

[15] S. T. Smith, Geometric optimization methods for adaptive filtering [PhD thesis], Harvard University, 1993.

[16] T. Inoue, H. Saruwatari, Y. Takahashi et al., "Theoretical analysis of musical noise in generalized spectral subtraction based on higher order statistics," IEEE Transactions on Audio, Speech, and Language Processing, vol. 19, no. 6, pp. 1770-1779, 2011. 

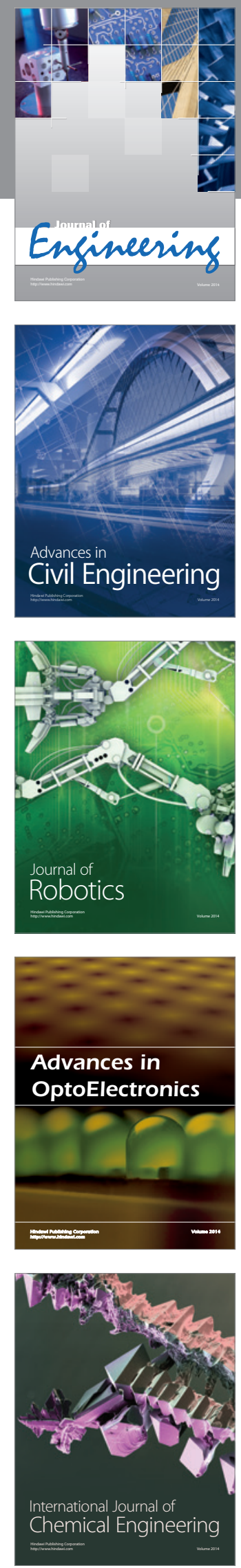

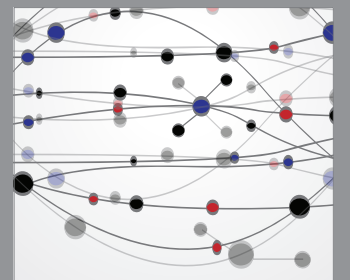

The Scientific World Journal
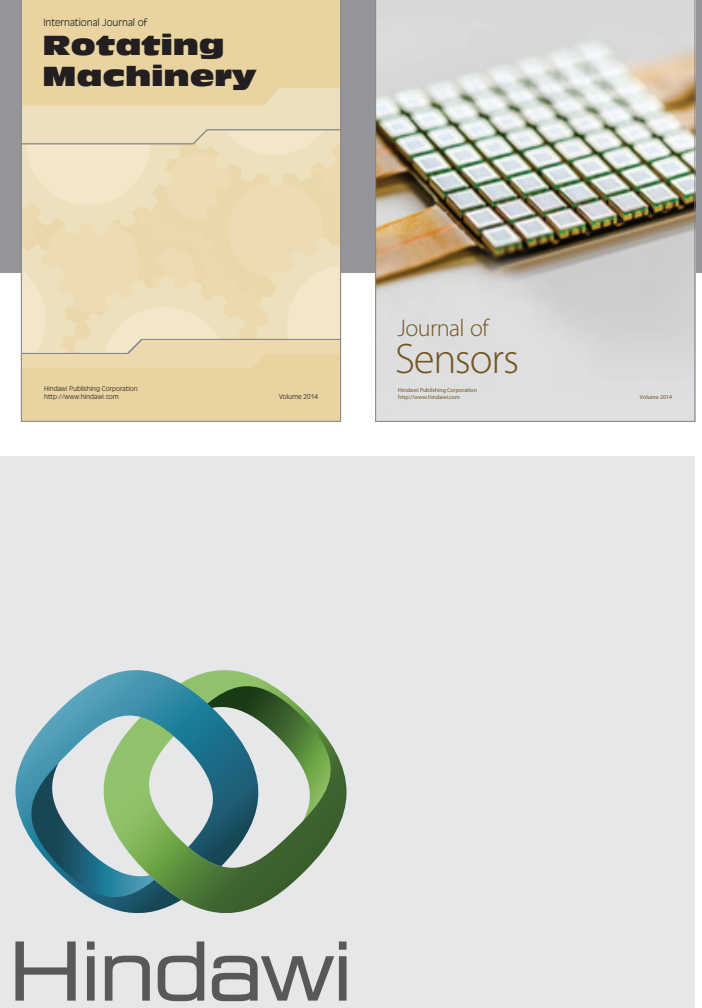

Submit your manuscripts at http://www.hindawi.com
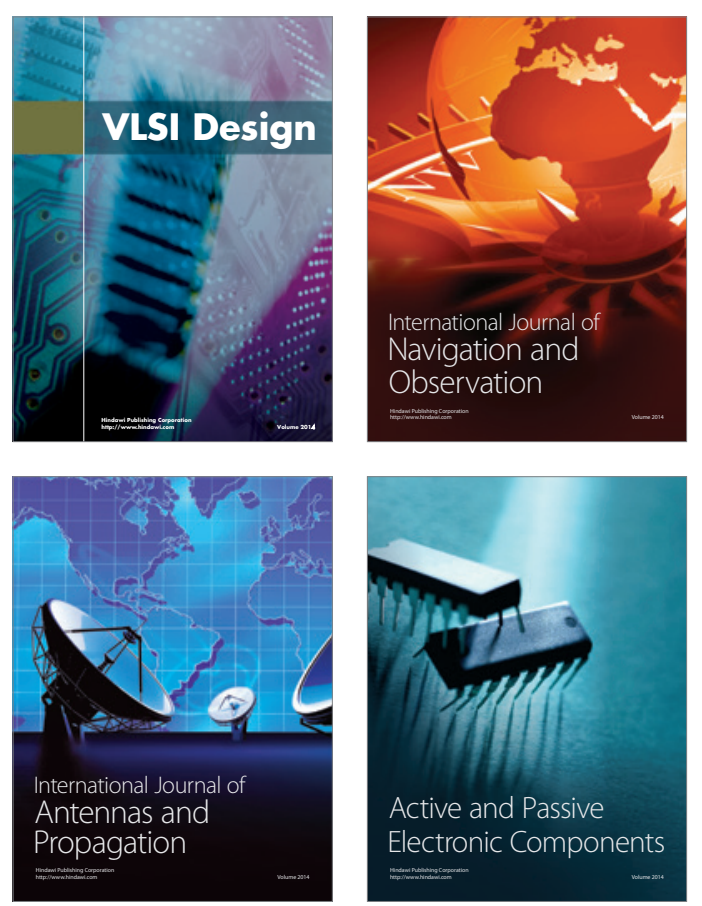
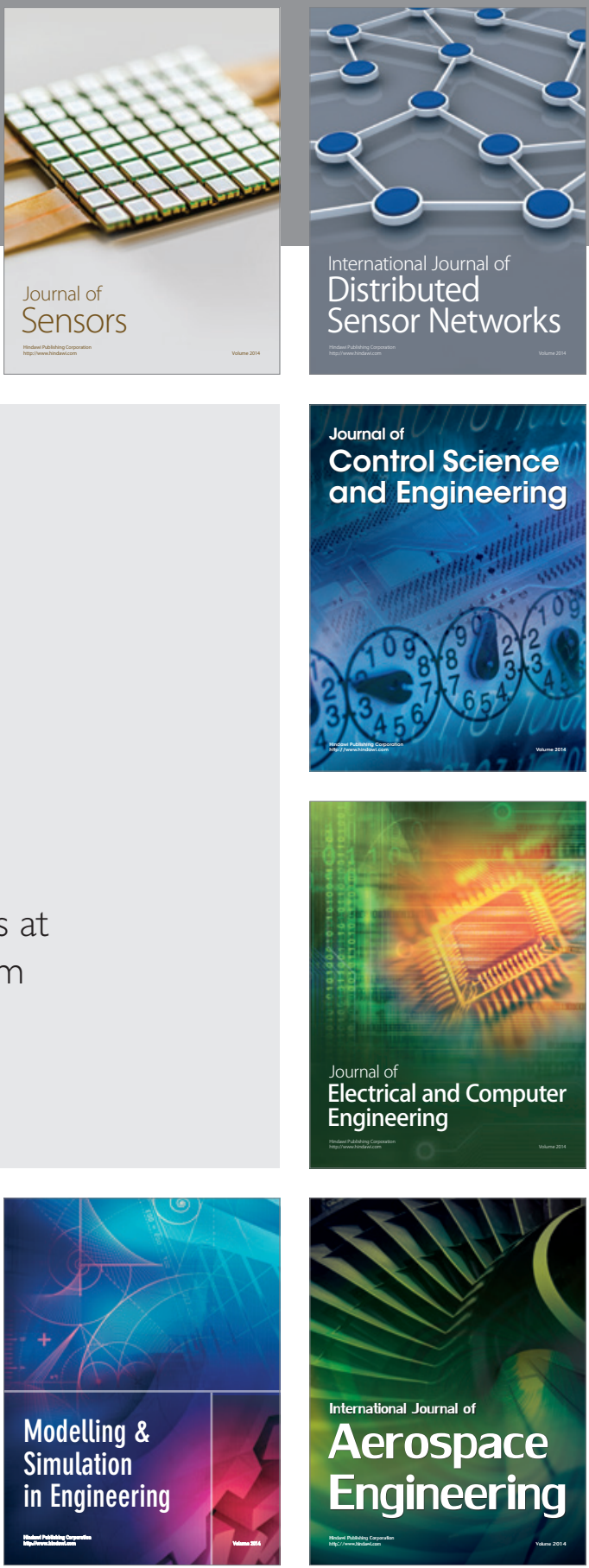

Journal of

Control Science

and Engineering
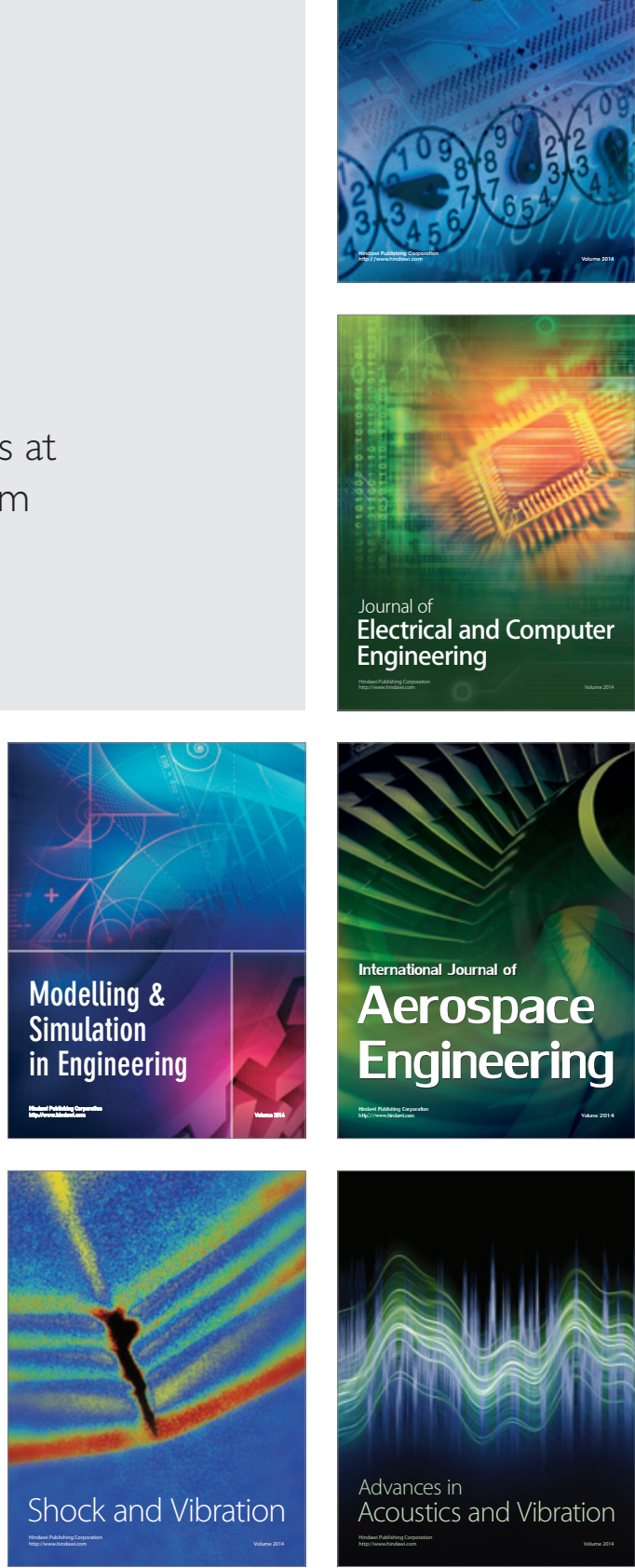